1998-02-01

\title{
Thermal equilibrium of warm clouds of charge with small aspect ratio
}

Deborah L. Paulson

Ross L. Spencer

ross_spencer@byu.edu

Follow this and additional works at: https://scholarsarchive.byu.edu/facpub

Part of the Astrophysics and Astronomy Commons, and the Physics Commons

\section{Original Publication Citation}

Paulson, Deborah L. and Ross L. Spencer."Thermal equilibrium of warm clouds of charge with small aspect ratio." Physics of Plasmas 5 (1998): 345-356.

\section{BYU ScholarsArchive Citation}

Paulson, Deborah L. and Spencer, Ross L., "Thermal equilibrium of warm clouds of charge with small aspect ratio" (1998). Faculty Publications. 652.

https://scholarsarchive.byu.edu/facpub/652

This Peer-Reviewed Article is brought to you for free and open access by BYU ScholarsArchive. It has been accepted for inclusion in Faculty Publications by an authorized administrator of BYU ScholarsArchive. For more information, please contact ellen_amatangelo@byu.edu. 


\title{
Thermal equilibrium of warm clouds of charge with small aspect ratio
}

\author{
Deborah L. Paulson and Ross L. Spencer ${ }^{a)}$ \\ Department of Physics and Astronomy, Brigham Young University, Provo, Utah 84602
}

(Received 12 August 1997; accepted 22 October 1997)

\begin{abstract}
Global thermal equilibrium computations are presented for non-neutral plasmas whose radial size is much larger than their axial thickness. Axial and radial density profiles are computed for both ideal and nonideal Penning trap fields. Simple results are obtained in the limits of both low and high central density. Comparison is made to the grid calculations of Mason et al. [Phys. Plasmas 3 (5), 1502 (1996)]. (C) 1998 American Institute of Physics. [S1070-664X(98)00402-9]
\end{abstract}

\section{INTRODUCTION}

When charged particles are loaded into a Penning trap, the initial density profile can be almost anything, depending on the details of the loading process. Slow transport, however, gradually causes the radius of the cloud to increase and drives the plasma toward global thermal equilibrium. If the plasma is cold enough that the Debye length is small compared to the cloud, the approach to global thermal equilibrium also causes the density profile to become uniform and the plasma shape to become a thin oblate spheroid. ${ }^{1}$

A warm plasma, however, behaves differently. Because of the kinetic energy of the particles, the plasma reaches a limit at which its thickness is on the order of the Debye length in the axial confining field. At this point, the plasma thickness can no longer decrease. As the radius grows, particle conservation requires the density to drop. This effect was observed in numerical simulations by Mason $^{2}$ et al. and is the subject of this paper.

Such pancake-like plasmas were studied in an experiment performed at the National Institute of Standards and Technology (NIST) in Boulder, Colorado by Weimer ${ }^{1}$ et al. In the experiment (consisting of about 43000 electrons that were reported to be held at a temperature of approximately 4 $\mathrm{K})$ the tenuous nature of the plasma made it necessary to find a nondestructive method of measuring its physical properties. The method used by Weimer et al. involved measuring the mode frequencies of the plasma and comparing them with the cold fluid theory of Dubin ${ }^{3}$ to find the plasma aspect ratio $\alpha$, defined as the ratio of the axial half-width $z_{p}$ to the radius $r_{p}$ of the plasma. This method worked reasonably well, but resulted in a systematic error of approximately $20 \%$ in the determination of $\alpha$ using different modes.

The Dubin theory is based on a zero-temperature, constant-density, non-neutral plasma confined axially by an electrostatic quadrupole potential. This externally imposed potential causes the cold plasma to assume a spheroidal shape. Given this uniform-density spheroidal plasma, Dubin used cold fluid theory to calculate the oscillation frequencies of the plasma. These frequencies are functions of the electrostatic confining field and the plasma aspect ratio.

Since the plasmas in Weimer's experiment were too warm for thermal effects to be negligible, a possible source

${ }^{a)}$ Electronic mail: spencerr@maxwell.byu.edu of the disagreement between the experimental results and Dubin's theory is the nonzero temperature. This possibility was studied in numerical simulations by Mason $^{2}$ et al. They found that the experimental plasmas should have had significant variations in density due to thermal effects. Mason et al. were able to study these thermal effects for some of Weimer's plasmas by using numerical grid calculations, but both computer memory and running time limitations made it difficult to handle the very thin plasmas of the experiment. In this paper a calculation that does not require a grid, and hence uses only small amounts of computer memory, will be described. The key idea of this calculation is to exploit the small aspect ratio of these thin plasmas to separate the calculation of the density profile into axial and radial parts. Because the calculation is somewhat analytic, it also provides interesting physical insights as well as analytic results in special limits.

Although Weimer's experiment served as the motivation for this study, there is one difficulty in comparing the results for a thin spheroid to Weimer's data. It was assumed in the experiment that the plasma remained spheroidal as the radius increased. Mason et al. demonstrated, however, that the nonideal components of the Penning trap field caused a distortion in the plasma shape, particularly at a small aspect ratio. For the sake of simplicity, most of the calculation presented here uses ideal confining fields. The more complicated nonideal fields encountered in experiments are discussed in the Appendices of this paper. In Sect. II we discuss global thermal equilibrium for a thin plasma confined by an external quadrupole field. Both axial and radial density profile calculations are presented. In Secs. III and IV we discuss the special cases of low and high central density, and in Sec. V, we conclude the paper. The same calculations for nonideal fields are discussed in Appendices A and B.

\section{GLOBAL THERMAL EQUILIBRIUM FOR THIN PLASMAS}

A plasma in global thermal equilibrium obeys the following form of Poisson's equation, ${ }^{4,5}$ where the potential $\phi$ $=\phi_{e}+\phi_{p}$ represents the sum of the external potential $\phi_{e}$ and the potential due to the plasma $\phi_{p}$,

$$
\nabla^{2} \phi=-\frac{q}{\epsilon_{0}} \overline{n e}-q \phi / k T+C r^{2},
$$


where $k$ is Boltzmann's constant, $q$ is the particle charge, and $\bar{n}$ is the central density. The constant $C$ is related to the canonical angular momentum and is given by $C=-(m / 2 k T) \omega\left(\omega+\omega_{c}\right)$, where $\omega$ is the rigid-rotor frequency of the global thermal equilibrium and where $\omega_{c}$ is the cyclotron frequency including the sign of the charge. ${ }^{4,5}$ Since we are not interested here in $\omega$, it is convenient to work only with $C$. It is assumed that the plasma is isolated in infinite space in the presence of an ideal external electrostatic quadrupole field. The boundary condition on $\phi_{p}$ at infinity is that it has the constant value that makes $\phi_{p}(0,0)=0$.

The external potential is the quadrupole field of a Penning trap, which can be written as

$$
\phi_{e}(r, z)=\frac{m}{2 q} \omega_{z}^{2}\left(z^{2}-\frac{1}{2} r^{2}\right),
$$

where $\omega_{z}$ is the Penning frequency, the axial bounce frequency of a single particle of mass $m$, and charge $q$ in the trap. This frequency is related to the density $n_{\text {cold }}$ of a cold spheroid of aspect ratio $\alpha$ by $^{6}$

$$
\omega_{z}=F(\alpha) \omega_{p},
$$

where

$$
\omega_{p}^{2}=\frac{q^{2} n_{\text {cold }}}{\epsilon_{0} m} .
$$

For low aspect ratio oblate spheroids (pancakes) ${ }^{6}$

$$
F^{2}(\alpha) \approx 1-\frac{\pi}{2} \alpha+2 \alpha^{2} .
$$

Cold spheroids will often be mentioned in the remainder of this paper. In fact, the point of most of this paper is to study what happens to a cold spheroid of radius $r_{p}$, halflength $z_{p}$, and central density $n_{\text {cold }}$ when its temperature is raised. This problem is quite difficult to solve for spheroids of an arbitrary aspect ratio, requiring the use of grid codes. ${ }^{5}$ (Note, however, that in the limit that the Debye length is small compared to the plasma thickness, Dubin has computed thermal corrections to cold equilibria. ${ }^{7}$ ) However, when the aspect ratio is small, it is possible to find an approximate separable form for the axial and radial density profiles for arbitrary values of the Debye length. These separate profile calculations will now be presented.

\section{A. Axial density profile: Thin approximation}

When the axisymmetric plasma is thin, the axial variation of the plasma potential in the neighborhood of the plasma is greater than the variation in the radial direction. This can be illustrated by solving the simple electrostatic problem of the distribution of potential across a uniformly charged infinitely thin disk with surface charge density $\sigma$ and radius $a$. The potential difference between the center and the outer edge is found to be

$$
\Delta \phi=\frac{\sigma a}{\epsilon_{0}}\left(1-\frac{2}{\pi}\right) .
$$

To apply this result to a thin plasma of density $n$ and thickness $z_{p}$, we use $\sigma \approx q n z_{p}$, so that

$$
\frac{1}{r} \frac{\partial}{\partial r} r \frac{\partial \phi_{p}}{\partial r} \approx\left(1-\frac{2}{\pi}\right) \frac{q n z_{p}}{\epsilon_{0} a},
$$

where the radial derivatives have simply been estimated by dividing by $a^{2}$.

The ratio of this part of $\nabla^{2} \phi_{p}$ to the full $\nabla^{2} \phi_{p}$ $=-q n / \epsilon_{0}$ is thus on the order of $z_{p} / a=\alpha$, which is small. In other words,

$$
\left|\frac{\partial^{2} \phi_{p}}{\partial z^{2}}\right| \gg\left|\frac{1}{r} \frac{\partial}{\partial r} r \frac{\partial \phi_{p}}{\partial r}\right| .
$$

It is easy to show that this result holds to an accuracy of order $\alpha$ for cold spheroids, and it was also checked for small aspect ratio thermal plasmas by using a grid code. ${ }^{5}$ This inequality is the basis of the thin approximation used throughout this paper and is synonymous with a low aspect ratio.

Using this thin approximation, the electrostatic problem can be separated into axial and radial parts. The axial equation is obtained simply by neglecting the radial part of $\nabla^{2}$ in Eq. (1).

To obtain an equation for the axial density profile, it is useful to describe the radial density profile in the plasma midplane with the dimensionless function $\beta(r)$ :

$$
n(r, 0)=\beta(r) n_{0},
$$

where the scale density $n_{0}$ is given by

$$
n_{0}=\frac{\omega_{z}^{2} \epsilon_{0} m}{q^{2}} .
$$

This density is nearly the same as $n_{\text {cold }}\left[n_{0}=F^{2}(\alpha) n_{\text {cold }}\right]$ but makes the resulting formulas simpler. It also simplifies comparisons with experiments because it depends only on the easily measured frequency $\omega_{z}$.

It is also useful to define the dimensionless potential $g(z)$ at each radius:

$$
g(z)=\frac{q \phi(r, z)}{k T}-\frac{q \phi(r, 0)}{k T},
$$

where $\phi$ is the total potential. Although $g(z)$ is actually a function of both $r$ and $z$, it is used to define the axial density profile at a given radius, so in order to simplify the notation it will be referred to simply as $g(z)$. With this definition for $g(z)$, the density profile can be written as

$$
n(r, z)=n_{0} \beta(r) e^{-g(z)} .
$$

In a further effort to make the equations dimensionless, we use a natural length scale $\lambda_{\mathrm{D} 0}$. This is a Debye length that depends on $n_{0}$ and on the temperature $T$ of the plasma:

$$
\lambda_{\mathrm{D} 0}=\sqrt{\frac{\epsilon_{0} k T}{q^{2} n_{0}}}=\sqrt{\frac{k T}{m \omega_{z}^{2}}} .
$$

The reason that this unusual definition for the Debye length is natural is that the axial confining electric field of the Penning trap is equivalent to the field of a uniform slab of constant charge density $n_{0}$ with a sign opposite to that of the confined particles. This is similar to the case of global thermal equilibrium in infinite cylinder geometry, ${ }^{4,5}$ where the confining magnetic field is equivalent to a uniform charge 


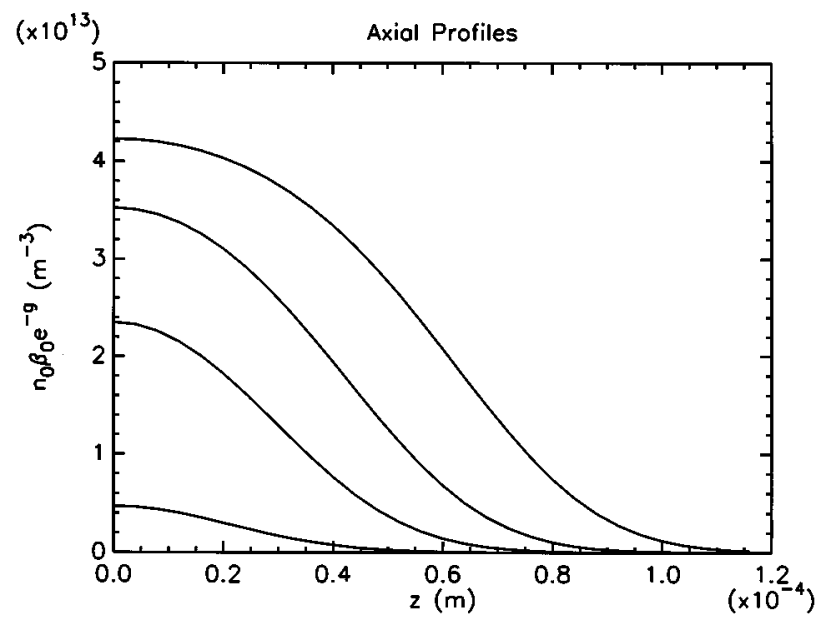

FIG. 1. A series of axial density profiles is shown for the following parameters: a 43000 electron plasma at $T=4 \mathrm{~K}$, confined in an ideal trap with $\omega_{z}=3.867 \times 10^{8} \mathrm{~s}^{-1}$. The shape of the axial profile depends on the value of $\beta_{0}=\beta(0)$. Profiles shown are for a scale density $n_{0}$ of $4.7 \times 10^{13} \mathrm{~m}^{-3}$. They correspond to $\beta_{0}$ values of $0.9,0.75,0.5$, and 0.1 .

density. The length $\lambda_{\mathrm{D} 0}$ is useful in the thin spheroid calculation discussed here because the Debye length defined in terms of the plasma central density varies both with plasma size and temperature, whereas $\lambda_{\mathrm{D} 0}$ only depends on temperature and the Penning trap field. This length also has physical meaning here, for we shall see later that in the limit of low plasma density the plasma thickness is of order $\lambda_{\mathrm{D} 0}$.

With this definition for $\lambda_{\mathrm{D} 0}$, we change to the dimensionless variable $\zeta=z / \lambda_{\mathrm{D} 0}$ and obtain an approximation to Eq. (1) at each radius in the form of an axial ordinary differential equation. This differential equation is derived using $\nabla^{2} \phi_{\text {ext }}=0$ and $\nabla^{2} \phi_{p} \approx \partial^{2} \phi_{p} / \partial z^{2}$. When Eq. (11) is used to evaluate $\partial^{2} \phi_{p} / \partial z^{2}$, the following differential equation for $g$ is obtained:

$$
\frac{d^{2} g}{d \zeta^{2}}=1-\beta(r) e^{-g}
$$

Because both the external and plasma potentials are chosen to vanish at the origin, it is clear that $g(0)=0$; and since both of these potentials are even functions of $z$, we have $g^{\prime}(0)=0$. These are the initial conditions for Eq. (14). Note that $\beta e^{-g}$ is the dimensionless axial density profile at a given radius.

This second-order ordinary differential equation can be solved numerically to obtain axial density profiles for various choices of $\beta(r)$. Figure 1 is a set of axial profiles at $r$ $=0$ obtained numerically for several values of $\beta(0)$ ranging from 0.1 up to 0.9 .

Note that in the limit of $\beta(r) \ll 1$, the density profile becomes

$$
n(r, z) \sim n_{0} \beta(r) e^{-(1 / 2) \zeta^{2}},
$$

so that in this limit the axial density profile is a Gaussian with a scale length of $\lambda_{\mathrm{D} 0}$. Hence, it is clear that the plasma can never be any thinner than about $\lambda_{\mathrm{D} 0}$. This low-density limit is discussed further in Sec. III.
Notice also that the solutions to Eq. (14) have the property that until $\beta$ is very close to unity, the width of the density profile $e^{-g}$ is characterized by $\lambda_{\mathrm{D} 0}$. As $\beta$ approaches unity, Eq. (14) implies a uniform axial density near the center of the plasma. This prediction that the plasma density becomes uniform as $\beta$ approaches 1 is in error, for the correct limiting value of $\beta$ obtained from Eq. (3) is $\beta$ $=1 / F^{2}(\alpha) \approx 1+\pi \alpha / 2$. This error, however, is only of order $\alpha$, as expected from using the thin approximation.

\section{B. Radial density profile}

In order to obtain the form for the midplane radial density profile $n(r, 0)$, we must first determine the midplane plasma potential, $\phi_{p}(r, 0)$ [see Eq. (1)]. Because of the thin approximation we have already made [Eq. (8)], it is difficult to use Eq. (1) in differential form to obtain $\phi_{p}(r, 0)$. Instead, we will use the electrostatic Green's function appropriate for $\phi_{p}(0,0)=0$,

$$
\begin{aligned}
\phi_{p}(r, 0)= & \int_{-\infty}^{\infty} \int_{0}^{\infty} 2 \pi\left[G\left(r, 0, r^{\prime}, z^{\prime}\right)\right. \\
& \left.-G\left(0,0, r^{\prime}, z^{\prime}\right)\right] q n_{0} \beta\left(r^{\prime}\right) e^{-g\left(z^{\prime}\right)} r^{\prime} d r^{\prime} d z^{\prime},
\end{aligned}
$$

where the cylindrical Green's function is given by

$$
G\left(r, z, r^{\prime}, z^{\prime}\right)=\frac{1}{4 \pi^{2} \epsilon_{0}} \sqrt{\frac{m}{r r^{\prime}}} K(m) .
$$

Here $K(m)$ is the complete elliptic integral of the first kind and

$$
m=\frac{4 r r^{\prime}}{\left(r+r^{\prime}\right)^{2}+\left(z-z^{\prime}\right)^{2}} .
$$

In the limit that the Debye length $\lambda_{\mathrm{D} 0}$ is small compared to the plasma radius, we again use the thinness of the plasma to treat it approximately as a disk with surface charge density $\sigma(r)=q_{-\infty}^{\infty} n(r, z) d z$. In this limit, $e^{-g}$ is highly localized so that it may be treated as an unnormalized delta function. The axial integration may then be performed to obtain

$$
\phi_{p}(r, 0)=\int_{0}^{\infty} \frac{\sigma\left(r^{\prime}\right)}{\epsilon_{0}} H\left(r, r^{\prime}\right) r^{\prime} d r^{\prime},
$$

where

$$
H\left(r, r^{\prime}\right)=2 \pi \epsilon_{0}\left[G\left(r, 0, r^{\prime}, 0\right)-G\left(0,0, r^{\prime}, 0\right)\right] .
$$

It is possible to obtain a fairly simple formula for $\sigma(r)$ by using the axial density profile calculation of Sec. II A as follows:

$$
\begin{aligned}
\sigma(r) & =q n_{0} \int_{-\infty}^{\infty} \beta(r) e^{-g} d z, \\
& =2 q n_{0} \lambda_{\mathrm{D} 0} \beta(r) \int_{0}^{\infty} e^{-g} d \zeta .
\end{aligned}
$$

This integral can be done more easily in the variable $g$. To obtain the integral in this form, multiply Eq. (14) by $g^{\prime}$ and integrate to obtain 


$$
g^{\prime}=\sqrt{2}\left[g+\beta(r)\left(e^{-g}-1\right)\right]^{1 / 2},
$$

so that the charge density becomes

$$
\sigma(r)=\sqrt{2} q n_{0} \lambda_{\mathrm{D} 0} \beta(r) \int_{0}^{\infty} \frac{e^{-g} d g}{\sqrt{g-\beta(r)\left(1-e^{-g}\right)}} .
$$

For simplicity, we define the function

$$
S(x)=\sqrt{2} \int_{0}^{\infty} \frac{e^{-g} d g}{\sqrt{g-x\left(1-e^{-g}\right)}} .
$$

With the dimensionless radial variable $\rho=r / r_{p}$, where $r_{p}$ is the maximum radial extent of the cold plasma, the surface charge density may be written as

$$
\sigma(\rho)=q n_{0} \lambda_{\mathrm{D} 0} \beta(\rho) S[\beta(\rho)] .
$$

Before proceeding further, it is useful to have available an approximate form for the function $S(x)$ valid for $x$ $<0.9$. An approximate form is given by the following:

$$
S(x) \approx \sqrt{2 \pi} \frac{1+p_{1} x}{1+p_{2} x}-2 \frac{1+p_{3}(1-x)}{1+p_{4}(1-x)} \ln (1-x),
$$

where the four parameters are best given by (for accuracy to about $0.01 \%$ )

$$
\begin{aligned}
& p_{1}=-0.82328, \quad p_{2}=-0.26673, \\
& p_{3}=1.92765, \quad p_{4}=1.40425 .
\end{aligned}
$$

Defining the dimensionless midplane plasma potential $h(\rho)$ by

$$
h(\rho)=\frac{q \phi_{p}(\rho, 0)}{k T},
$$

the electrostatic equation in the plasma midplane becomes

$$
h(\rho)=\frac{r_{p}}{\lambda_{\mathrm{D} 0}} \int_{0}^{\infty} \beta\left(\rho^{\prime}\right) S\left[\beta\left(\rho^{\prime}\right)\right] H\left(\rho, \rho^{\prime}\right) \rho^{\prime} d \rho^{\prime},
$$

where $\beta(\rho)$ depends on $h(\rho)$ through the form for the density given in Eq. (1):

$$
\beta(r)=\beta_{0} \exp \left(C r^{2}+\frac{1}{4 \lambda_{\mathrm{D} 0}^{2}} r^{2}-h(r)\right),
$$

and $\beta_{0}=\beta(0)$. This equation for $\beta(r)$ can be simplified by defining

$$
D=C r_{p}^{2}+\frac{r_{p}^{2}}{4 \lambda_{\mathrm{D} 0}^{2}}
$$

so that

$$
\beta(\rho)=\beta_{0} e^{D \rho^{2}-h(\rho)} .
$$

For fixed $\beta_{0}$ and $D$, Eqs. (29) and (32) can be solved simultaneously to obtain the radial density profile $\beta(\rho)$. In the calculation described here this is done by using successive substitution with under-relaxation on Eq. (29): ${ }^{5}$

$$
\begin{aligned}
h_{n+1}(\rho)= & (1-\epsilon) h_{n}(\rho)+\epsilon \frac{r_{p}}{\lambda_{\mathrm{D} 0}} \int_{0}^{\infty} \beta_{n}\left(\rho^{\prime}\right) \\
& \times S\left[\beta_{n}\left(\rho^{\prime}\right)\right] H\left(\rho, \rho^{\prime}\right) \rho^{\prime} d p^{\prime},
\end{aligned}
$$

where $h_{n}$ is the $n$th iterate and where $\epsilon$ is a small underrelaxation parameter on the order of $\lambda_{\mathrm{D} 0} / r_{p}$.

For specified values of $\beta_{0}$ and $D$, radial density profiles can be obtained from Eq. (33), but it is desirable to be able to vary the temperature and have the computed plasma equilibria maintain the same total number of particles $N$ and the same canonical angular momentum $p_{\theta}$ as a reference cold spheroid. Imposing these constraints determines $\beta_{0}$ and $D$, and their calculation will be discussed in the next section.

\section{Constraints}

It is interesting to compare this model to a cold spheroidal plasma to answer the question of what happens to the cold spheroid as its temperature is raised. If the temperature is raised without adding or removing particles or applying any torques, then both $N$ and $p_{\theta}$ must be conserved. In the limit of a small Larmor radius $\left(r_{L} \ll r_{p}\right), p_{\theta} \propto\left\langle r^{2}\right\rangle$. This limit is assumed in the following derivation.

For a cold spheroid, $N$ and $\left\langle r^{2}\right\rangle$ are given by

$$
N=\frac{4}{3} \pi r_{p}^{3} \alpha n_{\text {cold }} \text { and }\left\langle r^{2}\right\rangle=\frac{2}{5} r_{p}^{2} .
$$

Note that knowledge of these two quantities and the Penning frequency of the trap $\omega_{z}$ is sufficient to determine $r_{p}, z_{p}$, and $n_{0}$ through Eq. (34) together with Eqs. (3) and (4). In the remainder of this paper when $z_{p}$ appears, it is assumed that it was determined from $N,\left\langle r^{2}\right\rangle$, and $\omega_{z}$ in this way.

For a thermal density profile, integration over $\sigma(\rho)$ changes Eqs. (34) to the following constraint equations. Insisting that particle number be conserved and using $F(\alpha)$ $\approx 1$ yields

$$
\int_{0}^{\infty} \beta(\rho) S[\beta(\rho)] \rho d p=\frac{2 z_{p}}{3 \lambda_{\mathrm{D} 0}},
$$

where $z_{p}$ is the cold axial half-width such that $z_{p}=\alpha r_{p}$. Insisting on $\left\langle r^{2}\right\rangle$ conservation yields

$$
\frac{\int_{0}^{\infty} \beta(\rho) S[\beta(\rho)] \rho^{3} d \rho}{\int_{0}^{\infty} \beta(\rho) S[\beta(\rho)] \rho d \rho}=\frac{2}{5} .
$$

These two constraint equations determine the values of $\beta_{0}$ and $D$.

These constants are found by simultaneously solving both the electrostatic equations [Eqs. (29) and (32)] and the constraint equations [Eqs. (35) and (36)]. There are probably many ways to do this, but one successful algorithm will be described here. (1) Begin by iterating on Eq. (33) a few times. (2) Solve Eq. (36) for $D$ using a nonlinear zero finder, and then go back to (1), repeating (1) and (2) together until an adequate level of convergence is achieved. (3) This procedure is followed by an adjustment of $\beta_{0}$ based on writing Eq. (35) in the form

$$
\beta_{0, n+1}=\frac{2}{3} \beta_{0, n} \frac{z_{p}}{\lambda_{\mathrm{D} 0}} \frac{1}{\int_{0}^{\infty} \beta_{n}(\rho) S\left[\beta_{n}(\rho)\right] \rho d \rho},
$$



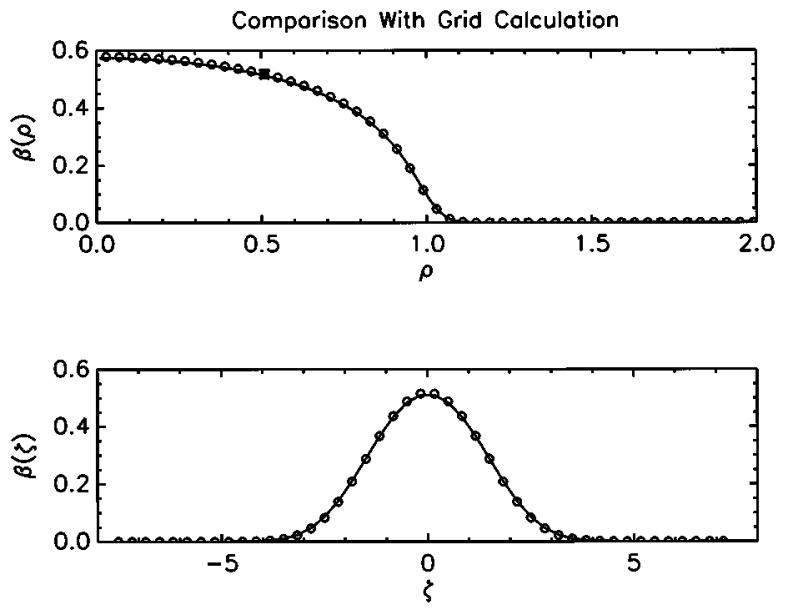

FIG. 2. A comparison with the memory intensive grid calculation of Mason et al. is shown. The case is a plasma consisting of 41888 electrons at $T$ $=2.1 \mathrm{~K}$ in an ideal trap with $\omega_{z}=5.5905 \times 10^{7} \mathrm{~s}^{-1}$. The cold spheroid density $n_{\text {cold }}$ is $1 \times 10^{12} \mathrm{~m}^{-3}$. The cold aspect ratio is 0.01 and the cold plasma radius is $0.01 \mathrm{~m}$. The top graph is a plot of the radial density profile. The open circles correspond to the data from the grid calculation, while the solid line represents the results from the calculation described in this paper. The lower graph is a plot of the axial density profile taken at the radius marked on the top graph with a solid square.

after which the algorithm returns again to (1). This entire procedure is continued until both of the constraints and the electrostatic equation are well satisfied. In difficult cases $\left(\beta_{0}\right.$ approaching unity) it is necessary to under-relax in step (3) as well.

To test the validity of this calculation, the results from it have been compared to the results of memory-intensive grid calculations. ${ }^{2,5}$ A global thermal equilibrium was computed on a grid with 100 radial grid points and 1200 axial grid points for a pancake with the same $N$ and $\left\langle r^{2}\right\rangle$ as a cold spheroid with an aspect ratio $\alpha=0.01$. The temperature corresponded to $\lambda_{\mathrm{D} 0} z_{p}=1$. A graphical comparison between the two methods for this case is shown in Fig. 2. The central density of the grid calculation was about $1 \%$ higher than that of the calculation described here and the radial and axial profiles agreed to the same precision. The error caused by using the thin approximation described in this paper is expected to be of order $\alpha$, which is consistent with the error in this and other comparisons. Since the thin approximation becomes more accurate as the aspect ratio of the plasma is decreased, the radial density profile calculation described in this section should be good to better than $1 \%$ for aspect ratios less than 0.01 .

\section{LOW CENTRAL DENSITY LIMIT}

It is possible to obtain the radial density profile in the special limit of low central density $\left(\beta_{0} \ll 1\right)$ using analytical methods. As $\beta_{0}$ approaches zero, Eq. (29) indicates that $h(\rho)$ also approaches zero so that the profile [see Eq. (32)] becomes a Gaussian,

$$
\beta(\rho)=\beta_{0} e^{D \rho^{2}} .
$$

Using this form in the constraint equations, Eqs. (35) and (36), and noting that $S(0)=\sqrt{2 \pi}, \beta_{0}$ and $D$ are determined to be

$$
\beta_{0}=\frac{10 z_{p}}{3 \sqrt{2 \pi} \lambda_{\mathrm{D} 0}} \text { and } D=-\frac{5}{2} .
$$

This result combined with the low-density limit for the axial profile [see Eq. (15)], yields a density profile that is double Gaussian,

$$
n(\rho, \zeta)=\beta_{0} n_{0} e^{-(1 / 2) \zeta^{2}-(5 / 2) \rho^{2}}
$$

This limit of small $\beta_{0}$ applies potentially to plasmas in an experiment such as Weimer's. As the plasma sits in the trap, the root-mean-square (rms) plasma radius grows due to transport. ${ }^{1}$ A cold plasma under these conditions would maintain its density and flatten $\left(z_{p} \rightarrow 0\right)$ to compensate for the increasing radius. When the plasma is warm, however, its thickness, or better, its thinness, is limited by the Debye length $\lambda_{\mathrm{D} 0}$. Because the plasma cannot become thinner than about $\lambda_{\mathrm{D} 0}$ as the radius grows, the central density is forced to drop in order to conserve the particle number. In time, the plasma would reach a state where the small $\beta_{0}$ limit describes its density profile. However, this limit is not reached until

$$
h(\rho) \ll D \rho^{2} .
$$

If $\beta_{0}$ is proportional to $z_{p} / \lambda_{\mathrm{D} 0}$ and $h(\rho)$ is proportional to $\beta_{0} r_{p} / \lambda_{\mathrm{D} 0}$ [see Eqs. (39) and (29)], then Eq. (41) implies that this limit is not reached until

$$
\frac{r_{p} z_{p}}{\lambda_{D 0}^{2}} \ll 1 .
$$

Numerical experiments with the equilibrium calculation described in Sec. II show that when the factor $r_{p} z_{p} / \lambda_{\mathrm{D} 0}^{2}$ $\leqslant 0.1$, the density profile is within a few percent of the approximate double Gaussian in Eq. (40).

The approach to this limit can be seen in Fig. 3. For instance, for case (d) in this figure we have $r_{p}$ $=\sqrt{2.5\left\langle r^{2}\right\rangle}=0.0632 \mathrm{~m}, \quad n_{\text {cold }} \simeq n_{0}=4.70 \times 10^{13} \mathrm{~m}^{-3}, \quad z_{p}$ $=5.46 \times 10^{-8} \mathrm{~m}$, and $\lambda_{\mathrm{D} 0}=6.37 \times 10^{-5} \mathrm{~m}$. Hence, for this case, $r_{p} z_{p} / \lambda_{\mathrm{D} 0}^{2}=0.85$, which is not small. Still, the profile shown in Fig. 3(d) is Gaussian to about $10 \%, D=-2.8$ (about $10 \%$ different from 2.5), and $\beta_{0}$ from Eq. (39) is 0.00114 , about $10 \%$ higher than the actual $\beta_{0}$.

\section{LOW-TEMPERATURE LIMIT}

In the opposite limit of the calculation, where $\beta_{0}$ $=1 / F^{2}(\alpha) \approx 1$, lies the cold plasma. At $T=0$, global thermal equilibrium gives a constant density spheroid. ${ }^{6,89}$ Following Bollinger $^{6}$ et al. we may therefore write for $\phi_{p}$,

$$
\phi_{p}(r, 0)=\frac{m}{4 q}\left[\omega_{z}^{2}-\omega_{p}^{2}\right] r^{2},
$$

which in the thin limit where $\alpha \ll 1$ becomes [using Eq. (3)]

$$
\phi_{p} \approx-\frac{\pi}{8} \frac{m z_{p}}{q r_{p}} \omega_{p}^{2} r^{2},
$$




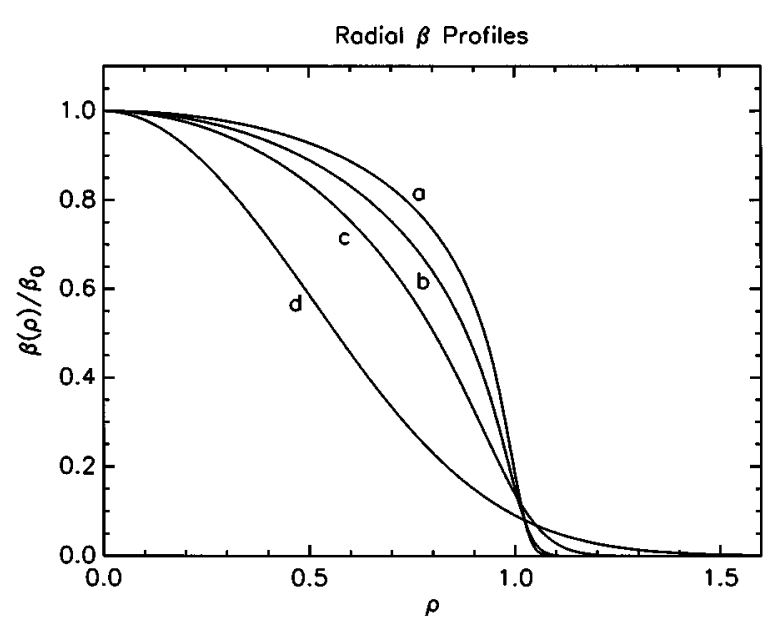

FIG. 3. A series of radial density profiles is shown for the following parameters: a 43000 electron plasma is confined in an ideal trap with $\omega_{z}=3.867$ $\times 10^{8} \mathrm{~s}^{-1}$. In this series, the root-mean-square (rms) plasma radius is varied from 1.6 to $40 \mathrm{~mm}$. The outer profile (a) corresponds to a rms plasma radius of $1.6 \mathrm{~mm}$ with $\beta_{0}=0.777$ at a temperature of $T=4 \mathrm{~K}$. At this small radius, the profile is approaching a uniform density step. The next profile (b) is for a rms plasma radius of $2.4 \mathrm{~mm}$ with $\beta_{0}=0.473$ also at $T=4 \mathrm{~K}$. The third profile (c) is for a rms plasma radius of $10 \mathrm{~mm}$ and has a low density: $\beta_{0}$ $=0.0364$, with $T=4 \mathrm{~K}$. The fourth profile $(d)$ is a special case to demonstrate the Gaussian limit. The rms plasma radius is $40 \mathrm{~mm}$, the temperature is $40 \mathrm{~K}$, and $\beta_{0}=0.00104$.

and the surface charge density of the thin cold spheroid of radius $r_{p}, \sigma(r)=q \int_{-\infty}^{\infty} n(r, z) d z$, can be written as

$$
\sigma(r)=2 q n_{\operatorname{cold}^{2}} z_{1} \sqrt{1-\frac{r^{2}}{r_{p}^{2}}} \text {. }
$$

Now imagine the temperature rising slightly. As long as $\lambda_{\mathrm{D} 0} \ll z_{p}$ the plasma approximately maintains its cold spheroid shape. But, as the temperature continues to rise so that $\lambda_{\mathrm{D} 0}$ approaches $z_{p}$, the axial density profile becomes rounded, as shown in Fig. 1. As this evolution occurs the constraint on $\left\langle r^{2}\right\rangle$ must be maintained. Because the radial density profile is proportional to $\exp \left[D \rho^{2}-h(\rho)\right]$ and both $D$ and $h(\rho)$ are very large for $\lambda_{\mathrm{D} 0} \approx z_{p} \ll r_{p}$ [see Eqs. (29) and (31)], to maintain $\left\langle r^{2}\right\rangle$ we must have $h(\rho) \approx D \rho^{2}$.

To find the surface charge density that could give rise to such a potential, we need only refer to the cold spheroid solution in Eqs. (44) and (45). These equations show that the charge density that would make a quadratic potential across a disk is

$$
\sigma(r) \propto \sqrt{1-\frac{r^{2}}{r_{p}^{2}}} .
$$

In fact, the constraint on $N$, which implies the same total charge for both thermal and cold spheroids, is all that is needed to see that the charge density for a thermal plasma with $\lambda_{\mathrm{D} 0} \approx z_{p}$ must be about the same as that for a cold spheroid, i.e.

$$
\sigma(\rho)_{\text {warm }} \approx \sigma(\rho)_{\text {cold }} .
$$

Such a condition may seem to be obvious, since this limit relies on $\lambda_{\mathrm{D} 0}$ being very small, i.e., the spheroid being fairly cold, but it is, in fact, an interesting result. If $h(\rho)$ $\approx D \rho^{2}$, then the charge density is nearly that of the cold spheroid. But in the low-temperature limit both $h$ and $D$ are very large, so even though $D \rho^{2} \approx h(\rho)$, this does not imply that $\beta(\rho)$ is flat or even nearly flat, since the difference between these two is the argument of an exponential. This means that even though $\sigma$ may stay approximately the same as it is for a cold spheroid, the density profile can change quite drastically. In fact, as the axial density profile is altered with an increase in temperature, the change in the radial density profile is the means by which $\sigma$ is able to remain the same.

This condition that $\sigma(\rho)_{\text {warm }}=\sigma(\rho)_{\text {cold }}$ requires that the potential $\phi_{p}(r)$ also be the same as in the case of the cold spheroid, giving

$$
h(\rho)=-\frac{\pi}{8} \frac{r_{p} z_{p}}{\lambda_{\mathrm{D} 0}^{2}} \rho^{2} .
$$

Finally, because $h(\rho) \approx D \rho^{2}$, we have

$$
D \approx-\frac{\pi}{8} \frac{r_{p} z_{p}}{\lambda_{\mathrm{D} 0}^{2}}
$$

valid when $|D|$ is large.

This approximation for $D$ will be accurate as long as the surface charge density is close to that of the cold spheroid. We have already assumed that $r_{p} z_{p} / \lambda_{\mathrm{D} 0}^{2} \gg 1$, so that $D$ must be very large. The question is now this: how large must $D$ be for this approximation (i.e., the equality of the surface charge densities) to remain valid?

One way to test the useful range of this approximation is to calculate $\beta(\rho)$ by iteration using $\sigma(\rho)$ as in Eq. (45). Here $\beta_{0}$ is first found by iteration using $\sigma_{0}=\sigma(0)$ [see Eqs. (25)(26)],

$$
\beta_{0, n+1}=\frac{2 z_{p}}{\lambda_{\mathrm{D} 0} S\left(\beta_{0, n}\right)} .
$$

This is then used to obtain $\beta(\rho)$,

$$
\beta_{n+1}(\rho)=\frac{\beta_{0} S\left(\beta_{0}\right)}{S\left[\beta_{n}(\rho)\right]} \sqrt{1-\rho^{2}} .
$$

Numerical comparisons between density profiles calculated in this way and density profiles from the calculation described in Sec. II show that this approximation is good to within a few percent if $D \leqslant-40$. This is when the $\sigma(\rho)$ profiles are very close to the cold spheroid $\sigma(\rho)$ profiles. Figure 4 shows a comparison between surface charge densities for a cold spheroid and a thermal spheroid with $D=-58.1$.

The approach to this limit can be seen in Fig. 3. For instance, for case (b) in this figure we have $r_{p}=\sqrt{2.5\left\langle r^{2}\right\rangle}$ $=0.00379 \mathrm{~m}, \quad n_{\text {cold }} \approx n_{0}=4.73 \times 10^{13} \mathrm{~m}^{-3}, \quad z_{p}=1.51$ $\times 10^{-5} \mathrm{~m}$, and $\lambda_{\mathrm{D} 0}=2.01 \times 10^{-5} \mathrm{~m}$. Hence, for this case, $r_{p} z_{p} / \lambda_{\mathrm{D} 0}^{2}=142$. The approximate $D$ from Eq. (49) is -55.6 , about $4 \%$ below its true value, and the value of $\beta_{0}$ obtained from the iteration indicated in Eq. (50) is 0.468 , about $1 \%$ below the actual value of 0.473 . This same limit is discussed for nonquadrupole confining fields in Appendices A and B.

Some final comments on the approach to $T=0$ are in order here. As the temperature drops toward zero, $\beta_{0}$ approaches 1 and $S\left(\beta_{0}\right)$ becomes logarithmically singular [see 


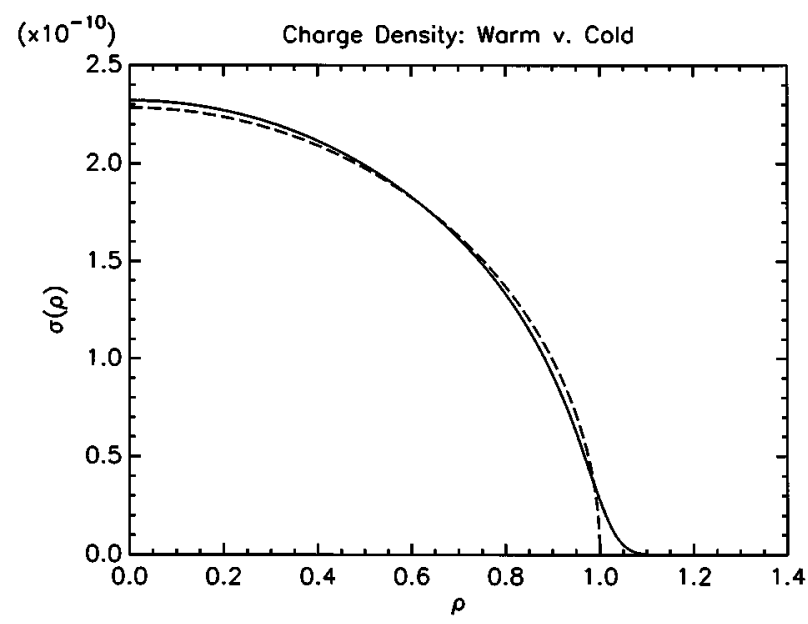

FIG. 4. A comparison between surface charge densities of a cold spheroidal plasma and a thermal plasma with $D=-58.1$, corresponding to the radial density profile $\beta(\rho)$ labeled (b) in Fig. 3, is shown. The cold $\sigma(\rho)$ corresponding to this case cuts off sharply at $\rho=1$.

Eq. (27)]. The presence of this singularity makes the iteration scheme indicated in Eqs. (50) and (51) not converge. However, if these two equations are solved by a more powerful algorithm, such as Newton's method, it is found that both $\beta_{0}$ and $\beta(\rho)$ approach unity, except right at the edge where $\sqrt{1-\rho^{2}}=0$. The correct limit is $1 / F^{2}(\alpha) \approx 1+\pi \alpha / 2$, so once again using the thin approximation gives an answer correct to order $\alpha$. This scheme is important computationally because the more complex algorithm described in Secs. II B and II C converges very poorly for $\beta_{0}>0.8$. Therefore, the only way to explore the approach to cold equilibrium is to use the approximate equations of this section.

\section{CONCLUSIONS}

When a cloud of charge in a Penning trap expands radially due to transport, it becomes thin axially. An important transition occurs when the plasma thickness approaches the length $\lambda_{\mathrm{D} 0}=\sqrt{k T / m \omega_{z}^{2}}$. When this thickness is reached, radial expansion cannot further reduce the thickness and thermal effects begin to dominate the equilibrium properties of the plasma. This can be an important effect, even in cryogenic systems. In the limit that the plasma thickness is much smaller than the plasma radius, an approximate theory that gives the equilibrium density as the product of an axial profile and a radial profile gives good results, both for ideal and nonideal electrostatic confining fields (see the Appendices). In this thin limit numerical calculations are required, but they are much faster than full two-dimensional calculations. In the limit that the Debye length is sufficiently small $\left(\lambda_{\mathrm{D} 0}\right.$ $\ll \sqrt{r_{p} z_{p}}$ ), it is found that even though the plasma density profile is quite different from the zero-temperature step-like profile, the line density profile $\int n d z$ is nearly the same as at zero temperature. In this case a simple nonlinear algebraic equation [see Eqs. (50)-(51)] may be solved to find density profiles, both for ideal and nonideal confining fields.

\section{ACKNOWLEDGMENTS}

The authors acknowledge helpful discussions with John Bollinger and Grant Mason, as well as the constructive comments of the reviewer of this paper.

\section{APPENDIX A: NONIDEAL CONFINING FIELDS}

A more general approach to this calculation is to consider a thermal plasma in a nonideal (nonquadrupole) confining field. To do so requires a knowledge of the external field in the midplane of the plasma. This field $\phi_{e}$ may be expressed using Legendre Polynomials:

$$
\phi_{e}(R, \Theta)=\sum_{n=1}^{\infty} C_{2 n} R^{2 n} P_{2 n}(\cos \Theta),
$$

where $(R, \Theta)$ are the radius and polar angle in spherical coordinates.

For a thin plasma, high-order axial terms such as $z^{4}, z^{6}, \ldots$, may be neglected. Newton's Second Law for a charged particle of mass $m$ and charge $q$ then implies that in this field,

$$
C_{2}=\frac{m}{2 q} \omega_{z}^{2}(0),
$$

so the external potential may be expressed as

$$
\phi_{e}=\frac{m}{2 q} \omega_{z}^{2}(r) z^{2}-\frac{m}{4 q} \omega_{z}^{2}(0) r^{2}[1+e(r)],
$$

where $\omega_{z}(r)$ is no longer a constant, but a function of $r$ in the midplane of the plasma, and $e(r)$ is a dimensionless parameter describing the higher-order corrections to the quadrupole field. For an ideal quadrupole, $\omega_{z}(r)=\omega_{z}(0)$ and $e(r)=0$. For the nonideal field, $\omega_{z}(r)$ and $e(r)$ are calculated from the trap parameters:

$$
\begin{aligned}
\omega_{z}^{2}(r) & =\omega_{z}^{2}(0)\left(1-\sum_{n=2}^{\infty} 2 n^{2} \frac{C_{2 n}}{C_{2}} P_{2 n}(0) r^{2 n-2}\right) \\
& \equiv \omega_{z}^{2}(0) \Omega(r), \\
e(r) & =\sum_{n=2}^{\infty} \frac{-2 C_{2 n}}{C_{2}} P_{2 n}(0) r^{2 n-2},
\end{aligned}
$$

where

$$
P_{2 n}(0)=\frac{(-1)^{n}(2 n) !}{[n !]^{2} 4^{n}} .
$$

Note that $\Omega(r)$ in Eq. (A4) is a dimensionless radial profile defined in order to simplify the notation in the equations that follow

$$
\Omega(r)=1-\sum_{n=2}^{\infty} 2 n^{2} \frac{C_{2 n}}{C_{2}} P_{2 n}(0) r^{2 n-2} .
$$

Once $\omega_{z}(r)$ and $e(r)$ are obtained from the $C_{n}$ coefficients, the calculation proceeds analogously to the perfect quadrupole calculation. 


\section{Modified equations}

Because there is no longer a cold spheroid to compare with, the plasma is specified by the quantities $N,\left\langle r^{2}\right\rangle$, and $T$. The main changes in the equations arise from the fact that $\omega_{z}$ and $\lambda_{\mathrm{D} 0}$ are no longer constants, but functions of radius, $\omega_{z}(r)$ and $\lambda_{\mathrm{D} 0}(r)$. For convenience, the scale density $n_{0}$ is given by

$$
n_{0}=\frac{\omega_{z}^{2}(0) \epsilon_{0} m}{q^{2}}
$$

and the Debye length $\lambda_{\mathrm{D} 0}(r)$ is given by

$$
\lambda_{\mathrm{D} 0}(r)=\sqrt{\frac{k T}{m \omega_{z}^{2}(r)}} .
$$

Defining the dimensionless coordinates, $\zeta$ and $\rho$,

$$
\zeta=\frac{z}{\lambda_{\mathrm{D} 0}(0)} \quad \text { and } \rho=\frac{r}{r_{\mathrm{rms}}},
$$

where $r_{\text {rms }}$ is the root-mean-square (rms) radial extent of the plasma, the rest of the calculation is relatively straightforward.

With the new external field, the axial equation [see Eq. (14)] becomes

$$
\frac{d^{2} g}{d \zeta^{2}}=\Omega(r)-\beta(r) e^{-g} .
$$

The surface charge density $\sigma(r)$ can then be written as

$$
\sigma(r)=q n_{0} \lambda_{\mathrm{D} 0}(r) \beta(r) S[\beta(r) / \Omega(r)] .
$$

For the radial equation [see Eq. (30)], we obtain

$$
\beta(r)=\beta_{0} \exp \left(C r^{2}+\frac{r^{2}}{4 \lambda_{\mathrm{D} 0}^{2}(0)}[1+e(r)]-h(r)\right),
$$

where $e(r)$ is calculated from the $C_{n}$ coefficients as indicated in Eq. (A5). Defining $D$ [see Eq. (31)] as

$$
D=C r_{\mathrm{rms}}^{2}+\frac{r_{\mathrm{rms}}^{2}}{4 \lambda_{\mathrm{D} 0}^{2}(0)},
$$

and $\epsilon(r)$ as

$$
\epsilon(r)=\frac{r^{2}}{4 \lambda_{\mathrm{D} 0}^{2}(0)} e(r),
$$

we have [see Eq. (32)]

$$
\beta(\rho)=\beta_{0} \exp \left[D \rho^{2}-h(\rho)+\epsilon(\rho)\right] .
$$

The electrostatic equation [see Eq. (29)] then becomes

$$
\begin{aligned}
h(\rho)= & \frac{r_{\mathrm{rms}}}{\lambda_{\mathrm{D} 0}^{2}(0)} \int_{0}^{\infty} \beta\left(\rho^{\prime}\right) S\left[\beta\left(\rho^{\prime}\right) / \Omega\left(\rho^{\prime}\right)\right] \\
& \times \lambda_{\mathrm{D} 0}\left(\rho^{\prime}\right) H\left(\rho, \rho^{\prime}\right) \rho^{\prime} d \rho^{\prime},
\end{aligned}
$$

and can be solved as before. are

The new constraint equations [see Eqs. (35) and (36)]

$$
N=2 \pi n_{0} r_{\text {rms }}^{2} \int_{0}^{\infty} \lambda_{\mathrm{D} 0}(\rho) \beta(\rho) S[\beta(\rho) / \Omega(\rho)] \rho d \rho
$$

and

$$
\frac{\int_{0}^{\infty} \lambda_{\mathrm{D} 0}(\rho) \beta(\rho) S[\beta(\rho) / \Omega(\rho)] \rho^{3} d \rho}{\int_{0}^{\infty} \lambda_{\mathrm{D} 0}(\rho) \beta(\rho) S[\beta(\rho) / \Omega] \rho d \rho}=1 .
$$

\section{Low-density limit for nonideal confining fields}

As before, we consider the special limit of low central density. Once again, we see that the dimensionless plasma potential $h(\rho)$ is small, and we can approximate $\beta(\rho)$ as

$$
\beta(\rho)=\beta_{0} \exp \left[D \rho^{2}+\epsilon(\rho)\right] .
$$

Using this form for $\beta(\rho)$ in the constraint equation for the number of particles yields

$$
\beta_{0}=\frac{N}{(2 \pi)^{3 / 2} n_{0} r_{\mathrm{rmS}}^{2} \int_{0}^{\infty} \lambda_{\mathrm{D} 0}(\rho) \exp \left[D \rho^{2}+\epsilon(\rho)\right] \rho d \rho} .
$$

Unfortunately, this equation is more complicated than its analog in the ideal quadrupole field. It is, however, possible to solve it numerically after using the angular momentum constraint and a nonlinear zero finder to solve for $D$. Already, however, one important feature of nonuniform external fields can be seen in Eq. (A20). If the nonideal terms in $\epsilon(\rho)$ are positive, then at large radius they will overcome the $D \rho^{2}$ term, giving a global thermal equilibrium state that is unconfined. This is discussed further in Sec. 4 of this appendix.

\section{Low-temperature limit for nonideal confining fields}

The same argument made in Sec. IV holds for the case of the nonideal confining field, so that once again we find

$$
\sigma(\rho)_{\text {warm }} \approx \sigma(\rho)_{\text {cold }},
$$

for sufficiently small $\lambda_{\mathrm{D} 0}(\rho)$. As before, the limit of low temperature implies that the argument of the exponent in Eq. (A16) is approximately zero,

$$
D \rho^{2}-h(\rho)+\epsilon(\rho) \approx 0,
$$

or

$$
h(\rho) \approx D \rho^{2}+\epsilon(\rho) .
$$

This means that the plasma potential is no longer proportional to $\rho^{2}$, but has contributions from all even powers of $\rho$. The charge density $\sigma(\rho)_{\text {cold }}$ that would produce such a potential is calculated in Appendix B.

By combining Eq. (A22) with the constraints on $N$ and $\left\langle r^{2}\right\rangle$, it is possible to determine $D$ numerically and to perform the following iteration in analogy with Eq. (51) to determine $\beta(\rho)$ :

$$
\beta_{n+1}(\rho)=\frac{\sigma(\rho)_{\text {cold }}}{q n_{0} \lambda_{\mathrm{D} 0}(\rho) S\left[\beta_{n}(\rho) / \Omega(\rho)\right]},
$$




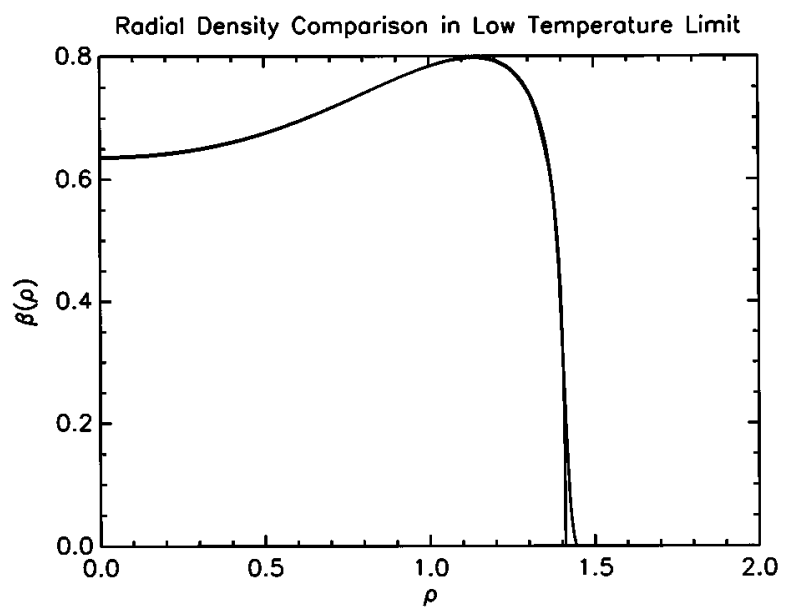

FIG. 5. A comparison between the radial density profile $\beta(\rho)$ from the low-temperature limit iteration [Eq. (A25)] and $\beta(\rho)$ from the full electrostatic calculation is shown. The iterated profile cuts off at a $\rho$ of approximately 1.40, while the profile from the full calculation cuts off at a $\rho$ of 1.44 . The profile corresponds to a plasma with 31592 electrons held at $T=1 \mathrm{~K}$ having a $r_{\text {rms }}$ of $1.9 \times 10^{-3} \mathrm{~m}$. See Fig. 6 in Appendix B for a comparison of $\sigma(\rho)_{\text {warm }}$ to $\sigma(\rho)_{\text {cold }}$ for this case.

valid for low temperatures $\left(\beta_{0} \geqslant 0.5\right)$. Using this iteration at different values of $\rho$, it is possible to obtain the $\beta(\rho)$ profile in this limit without performing the difficult electrostatic integration in Eq. (A17).

Figure 5 shows a comparison between the radial density profiles calculated using Eq. (A25) and the full electrostatic calculation. The case shown is for a temperature of $T$ $=1 \mathrm{~K}$, with 31592 electrons in a nonideal confining field. The radius $r_{\text {rms }}$ was $1.9 \times 10^{-3} \mathrm{~m}$, and $\beta_{0}$ was 0.637 . The coefficients of the external field as determined by analyzing the vacuum field from the code of Ref. 5 were

$$
\begin{aligned}
C_{2}, C_{4}, \cdots= & -4.1220 \times 10^{5}, \quad-8.6641 \times 10^{7}, \\
& 6.5565 \times 10^{13},-3.2567 \times 10^{17}, \\
& -3.7686 \times 10^{22}, \quad 5.4069 \times 10^{27} \ldots .
\end{aligned}
$$

Agreement is good to better than $1 \%$ for most of the profile. Beyond the radius of the equivalent cold plasma, however, the approximate iteration of course fails to give the correct nonzero value for $\beta$ in the thermal tail of the density distribution.

\section{Possible effects of nonideal fields on radial confinement}

It is interesting to note that Weimer et al. discovered that a large $\left|C_{4}\right|$ actually seemed to slow the radial expansion of the plasmas, making it possible to measure the mode frequencies for a longer time. ${ }^{1}$ Equations (1), (A3), and (A5) indicate that $C_{4}$ can significantly affect the radial density profile. For instance, if $C_{4}$ and $C_{2}$ are of the same sign, the non-ideal part of the external field can help drive the density toward zero at large radius. For $C_{4}$ and $C_{2}$ of opposite sign, however, this same non-ideal part of the field tends to make the density profile become infinite at large radius.
This effect is most easily seen in the low-density limit [see Eq. (A20)]. At radii for which the nonideal corrections in the function $\epsilon(\rho)$ are not small, it will dominate the behavior of $\beta(\rho)$. [Note: strictly speaking, $\epsilon(\rho)$ always dominates at large radius, but real systems are of finite size, so only radii less than the system radius should be considered.) If $\epsilon(\rho)$ is negative then it will cause the density profile to cut off sharply, making the global thermal equilibrium state toward which transport is driving the system better confined radially. When this effect is important, the term $D \rho^{2}$ in Eq. (A20) must tend to become positive to keep the particle count constant. This effect can give rise to hollow density profiles, as discussed in Appendix B and in Mason ${ }^{2}$ et al.

This occurs typically when $C_{4}$ has the same sign as $C_{2}$ [see Eq. (A5)], although the higher-order terms often matter too. If, however, $\epsilon(\rho)$ is positive $\left(C_{4}\right.$ and $C_{2}$ of opposite sign, typically), the density profile will tend to blow up at large radius, possibly giving an unconfined global thermal equilibrium state if this effect is important at the radius of the confining conductors. In practice, this would probably mean that evolution toward global thermal equilibrium would tend to make the plasma concentrated near the outer ring of the trap in this case.

This influence of the external field has been seen in numerical grid experiments we have performed in which a real Penning trap geometry was used to solve for the vacuum (external) field for various trap tunings (different guard ring voltages; see Weimer ${ }^{1}$ et al.). By solving for the potential and subtracting away the ideal components of the field, we found the higher-order contributions to the field. Then, by plotting $\exp \left[-q \overline{\phi_{e}} / k T\right]$, where $\overline{\phi_{e}}$ represents only the higherorder contributions, it is easily determined whether the trap will provide radial confinement or radial deconfinement for the plasma in global thermal equilibrium. Using the same geometry as in the experiment by Weimer et al., we found that with the guard ring voltage at $0.0 \mathrm{~V}$, the higher-order terms in the external potential were such as to cause deconfinement, but by raising the voltage on the ring, making $C_{4}$ larger and of the same sign as $C_{2}$, radial confinement was enhanced. This effect was clear in the numerical experiments, but it was not possible to duplicate the numerical values of $C_{4}$ given in Weimer et al. This may be due to a difference between the experimental apparatus and the numerical version of it used in the grid calculation, or perhaps due to different ways of calculating $C_{4}$. It would be nice to perform another experiment to test these ideas.

\section{APPENDIX B: COLD EQUILIBRIA IN NONIDEAL FIELDS}

To fully explore the problem of what thin thermal plasma equilibria look like when the confining field is not ideal, it is necessary to solve the equilibrium problem in such fields at zero temperature. As $T$ approaches zero, Eq. (1) requires that

$$
\phi_{p}(r, 0)=-\phi_{e}(r, 0)+\frac{C k T}{q} r^{2},
$$


which means that the form of the potential produced by the plasma is known. (The constant $C$ must be determined selfconsistently by the radial size of the plasma, but the $r$ dependence of the term in which it appears is known.) Because the plasma equilibria are assumed to be thin, the cold equilibrium problem can be stated this way: given a known distribution of potential $\phi_{p}(r)$ across a disk of radius $a$, what surface charge density distribution $\sigma(r)$ produces it? This problem can be approached by assuming that the desired $\phi_{p}$ is represented by a power series in $r^{2}$ and by finding the corresponding $\sigma$ for each power. Hence, the problem of cold thin plasma in global thermal equilibrium can be solved if the following electrostatic problem can be solved: given a charged disk of radius $a$, what surface charge distribution $\sigma(r)$ will produce a potential $\phi(r)=V_{0}(r / a)^{2 n}$ on the surface of the disk? The solution of this problem is the main subject of this Appendix, but physical consequences are discussed at the end of the Appendix. [Remember that throughout the paper it is assumed that $\phi(0)=0$, which means that the constant potential at the center of the disk is always subtracted away.]

\section{Surface charge distributions for cold equilibria}

To solve for the surface charge distribution, the axisymmetric Green's function used in Sec. II B is inconvenient because it involves an elliptic integral. It is easier to obtain an analytic connection between $\sigma(r)$ and $\phi(r)$ by performing a two-dimensional integral over the surface of the disk using a cylindrical coordinate system $(\rho, \theta)$ whose origin is at $x=r$. Using this coordinate system, the distance $r^{\prime}$ from the center of the disk is given by

$$
r^{\prime}=\sqrt{r^{2}+2 \rho r \cos \theta+\rho^{2}}
$$

and the equation of the edge of the disk is

$$
\rho(\theta)=-r \cos \theta+\sqrt{a^{2}-r^{2} \sin ^{2} \theta} .
$$

The potential at a distance $r$ from the center of the disk is then given by

$$
\phi(r)=\frac{1}{4 \pi \epsilon_{0}} \int_{0}^{2 \pi} \int_{0}^{\rho(\theta)} \sigma\left(r^{\prime}\right) d \rho .
$$

We already know the answer in the case of $\phi(r) \propto r^{2}$ from Eqs. (44)-(45) in Sec. IV:

$$
\sigma(r)=\sigma_{0} \sqrt{1-r^{2} / a^{2}} \Rightarrow \phi(r)=-\frac{\pi \sigma_{0} a}{16 \epsilon_{0}}\left(\frac{r}{a}\right)^{2} .
$$

In the process of obtaining this result from Eq. (B4) an integral of a special type is encountered, which leads to the solution of the desired problem for all powers of $r^{2}$. This integral is

$$
\begin{aligned}
\int X^{n+1 / 2} d \rho= & \frac{(2 n+2) !}{[(n+1) !]^{2}(4 k)^{n+1}}\left(\frac{k(2 C \rho+B) \sqrt{X}}{C}\right. \\
& \left.\times \sum_{r=0}^{n} \frac{r !(r+1) !}{(2 r+2) !}(4 k X)^{r}+\int \frac{d \rho}{\sqrt{X}}\right),
\end{aligned}
$$

where

$$
X(\rho)=A+B \rho+C \rho^{2} ; \quad k=\frac{4 C}{4 A C-B^{2}} .
$$

It will be shown that this integral can be applied to the problem given by Eq. (B4) by choosing surface charge distributions proportional to $\left(a^{2}-r^{2}\right)^{n+1 / 2}$ (note that other choices may be possible), and choosing

$$
X=a^{2}-r^{\prime 2}=a^{2}-r^{2}-2 r \rho \cos \theta-\rho^{2} .
$$

This means that $X$ vanishes on the edge of the disk, i.e., $X[\rho(\theta)]=0$ [see Eq. (B3)], and that $k=1 /\left(a^{2}-r^{2} \sin ^{2} \theta\right)$. The integral in Eq. (B6) can now be used to perform the $\rho$ integration in Eq. (B4). In this integral the following simplifications occur: (1) the complicated term containing the sum on $r$ vanishes at the upper limit because $X$ vanishes there, and (2) the lower limit gives a function that integrates to 0 when the $\theta$ integral in Eq. (B4) is performed. The remaining term can be integrated:

$$
\int_{0}^{\rho(\theta)} \frac{d \rho}{\sqrt{X}}=-\left.\sin ^{-1}\left(\frac{-2 \rho-2 r \cos \theta}{\sqrt{4\left(a^{2}-r^{2} \sin ^{2} \theta\right)}}\right)\right|_{0} ^{\rho(\theta)} .
$$

The upper limit is simply $\pi / 2$ while the lower limit gives a function of $\theta$ that is annihilated by the $\theta$ integral in Eq. (B4). Hence, the following useful integration formula related to Eq. (B4) is obtained:

$$
\begin{aligned}
\int_{0}^{2 \pi} d \theta \int_{0}^{\rho(\theta)} X^{n+1 / 2} d \rho= & \frac{(2 n+2) !}{[(n+1) !]^{2}(4)^{n+1}} \frac{\pi}{2} \\
& \times \int_{0}^{2 \pi}\left(a^{2}-r^{2} \sin ^{2} \theta\right)^{n+1} d \theta .
\end{aligned}
$$

The remaining integral can be performed by using the binomial expansion:

$$
\begin{aligned}
\int_{0}^{2 \pi} & \left(a^{2}-r^{2} \sin ^{2} \theta\right)^{n+1} d \theta \\
= & 2 \pi a^{2(n+1)} \sum_{m=0}^{(n+1)} B_{m}\left(\begin{array}{c}
n+1 \\
m
\end{array}\right)\left(\begin{array}{c}
r \\
a
\end{array}\right)^{2 m},
\end{aligned}
$$

where $\left(\begin{array}{l}n+1 \\ m\end{array}\right)$ is the binomial coefficient and

$$
B_{m}=\frac{(-1)^{m}(2 m) !}{(m !)^{2} 4^{m}}=P_{2 m}(0),
$$

and $P_{m}(x)$ is the Legendre polynomial.

Using this result, we may now write down the potential $\phi(r)$ produced by the family of surface charge densities that are proportional to $\left(a^{2}-r^{2}\right)^{n+1 / 2}$ :

$$
\begin{aligned}
\sigma(r)= & \sigma_{n}\left(1-r^{2} / a^{2}\right)^{n+1 / 2} \\
& \Rightarrow \phi(r)=\frac{\pi}{4} \frac{\sigma_{n} a}{\epsilon_{0}}\left|B_{n+1}\right| \sum_{m=0}^{n+1} B_{m}\left(\begin{array}{c}
n+1 \\
m
\end{array}\right)\left(\begin{array}{c}
r \\
a
\end{array}\right)^{2 m} .
\end{aligned}
$$

Now we are quite close to solving the problem of finding what surface charge distribution makes the potential $V_{o}(r / a)^{2 n}$ across its surface, because we have found a family 
of surface charge densities that make potentials that are polynomials in $(r / a)^{2}$. Note from Eq. (B13) that any given value of $n$ produces a polynomial $\phi(r)$ whose highest power is $(r / a)^{2(n+1)}$. Hence, to produce $\phi(r)=V_{0}(r / a)^{2 n}$ we may choose $\sigma_{n-1}$ to match it in Eq. (B13), then find the proper values of $\sigma_{m}$ for $0 \leqslant m<n-1$ to eliminate the lower powers of $(r / a)^{m}$ in the expression for $\phi(r)$ in Eq. (B13). This linear algebra problem may be solved to obtain the following:

$\phi(r)=V_{0}(r / a)^{2 n}$ is produced by $\sigma(r)=\frac{4 \epsilon_{0} V_{0}}{\pi a} s_{n}(r / a)$,

where the function $s_{n}(x)$ is given by

$$
s_{n}(x)=\sum_{m=0}^{n-1} \frac{(-1)^{m+1}}{\left|B_{n} B_{m+1}\right|}\left(\begin{array}{c}
n \\
m+1
\end{array}\right)\left(1-x^{2}\right)^{m+1 / 2} .
$$

Equations (B14) and (B15) may now be used to solve the problem of what $\sigma(r)$ corresponds to thin global thermal equilibrium as $T$ approaches zero. It will be assumed that the external confining field is known through the coefficients $C_{2 n}$ in the expansion

$$
\phi_{e}(R, \Theta)=\sum_{n=1}^{\infty} C_{2 n} R^{2 n} P_{2 n}(\cos \Theta)
$$

where $(R, \Theta)$ are the radius and polar angle in spherical coordinates. It is also assumed that the total number of particles $N$ in the equilibrium is known, as well as the rms plasma radius $r_{\text {rms }}$. As in the ideal case, the $r^{2}$ term in $\phi_{e}$ and the angular momentum term involving $C$ in Eq. (B1) combine together to give the following form for $\phi_{p}(r)$ at $z=0$ :

$$
\phi_{p}(r)=\vec{V} \frac{r^{2}}{a^{2}}-\sum_{n=2}^{\infty} C_{2 n} a^{2 n} P_{2 n}(0)\left(\frac{r}{a}\right)^{2 n}
$$

where $\bar{V}$ corresponds to the constant $D$ defined in Sec. II B and where $a$ is the as-yet-unknown outer radius of the cold plasma. These two constants are to be determined by the particle number and the rms plasma radius.

To make the connection between $(V, a)$ and $\left(N, r_{\text {rms }}\right)$, and to find $\sigma(r)$, we use Eqs. (B14), (B15), and (B17):

$$
\sigma(r)=\frac{4 \epsilon_{0}}{\pi a}\left(\overline{V s}_{1}(r / a)-\sum_{n=2}^{\infty} C_{2 n} a^{2 n} P_{2 n}(0) s_{n}(r / a)\right) .
$$

The particle number and $r_{\text {rms }}$ may now be determined by taking radial moments of $\sigma(r)$ as follows:

$$
\begin{aligned}
& N=\frac{2 \pi}{q} \int_{0}^{a} \sigma(r) r d r ; \quad P=\frac{2 \pi}{q} \int_{0}^{a} \sigma(r) r^{3} d r ; \\
& r_{\mathrm{rms}}=\sqrt{P / N} .
\end{aligned}
$$

Since Eq. (B18) gives an explicit form for $\sigma(r)$, these integrals may be performed to obtain the following formulas for $N$ and $P$ :

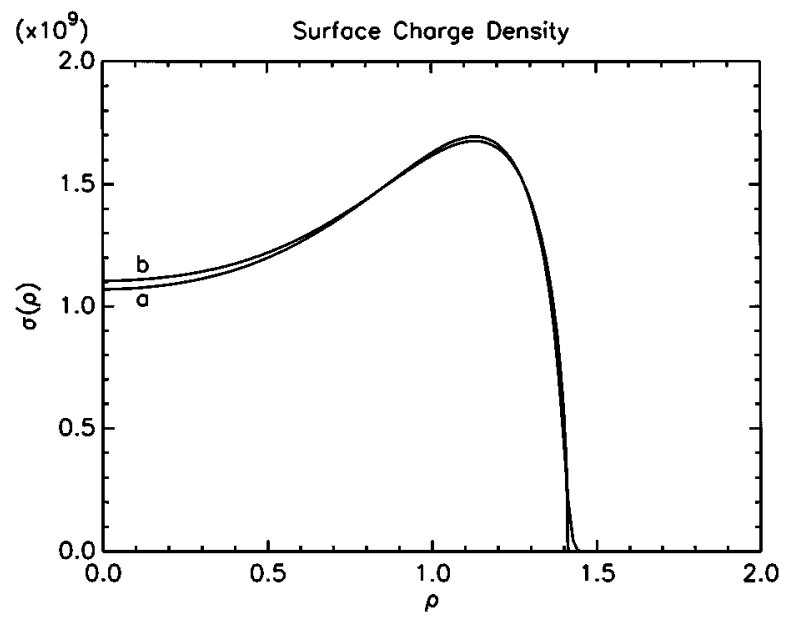

FIG. 6. The computed surface charge densities from the $T=0$ calculation of Appendix B (b) and from a low-temperature $(T=1 \mathrm{~K})$ grid calculation (a) are compared. The case is a plasma consisting of 31592 electrons in a nonideal trap.

$$
\begin{aligned}
N= & \frac{8 a \epsilon_{0}}{q}\left(-\bar{V} \frac{1}{3 B_{1}^{2}}\right. \\
& \left.+\sum_{n=2}^{\infty} \sum_{m=0}^{n-1} C_{2 n} a^{2 n} \frac{(-1)^{m+n}\left(\begin{array}{c}
n \\
m+1
\end{array}\right)}{\left|B_{m+1}\right|(2 m+3)}\right), \\
P= & \frac{16 a^{3} \epsilon_{0}}{q}\left(-\bar{V} \frac{1}{15 B_{1}^{2}}\right. \\
& \left.+\sum_{n=2}^{\infty} \sum_{m=0}^{n-1} C_{2 n} a^{2 n} \frac{(-1)^{m+n}\left(\begin{array}{c}
n \\
m+1
\end{array}\right)}{\left|B_{m+1}\right|(2 m+3)(2 m+5)}\right) .
\end{aligned}
$$

Unfortunately, this is as far as analysis can take us. Even if only one nonideal term $\left(C_{4}\right)$ is included, a seventh-order polynomial equation must be solved to determine $a$. Therefore, to determine $V$ and $a$ it is necessary to solve Eqs. (B19) numerically. Note, however, that if $a$ is known, then the equation for $P$ may be ignored and $V$ may easily be obtained from Eq. (B20).

Figure 6 shows how this calculation compares with a plasma equilibrium calculation done with the finitedifference code described in Ref. 5. The calculation was carried out in the geometry of Weimer's experiment, ${ }^{1}$ as used in the calculations of Mason ${ }^{2}$ et al. The voltage on the guard ring was $+8.7 \mathrm{~V}, \omega_{z}=3.81 \times 10^{8} \mathrm{~s}^{-1}$ and the coefficients of the external field are as given in Appendix A [see Eq. (A26)]. The plasma consists of 31592 electrons at a temperature of $1 \mathrm{~K}$, with $r_{\mathrm{rms}}=1.9 \times 10^{-3} \mathrm{~m}$. The calculation was performed on a grid with 300 radial points and 1000 axial points. The two calculations should not agree exactly because of the nonzero temperature in the grid calculation, but they are quite similar. 


\section{Cold plasma shapes in presence of nonzero $C_{4}$}

One interesting application of this result is found in the calculation of the plasma shape at $T=0$. If the plasma is thin enough that the nonideal analog of the approximation $F(\alpha)$ $\approx 1$ is valid [see Eq. (5)], then at each radius a $T=0$ plasma would condense to the cold density,

$$
n_{\text {cold }}(r)=\frac{\omega_{z}^{2}(r) \epsilon_{0} m}{q^{2}},
$$

where $\omega_{z}^{2}(r)$ is given in Eq. (A4). However, if both $n(r)$ and $\sigma(r)$ are known, then the axial half-width $z_{p}(r)$ is easily determined to be

$$
z_{p}(r)=\frac{\sigma(r)}{2 q n_{\text {cold }}(r)} .
$$

This makes it possible to calculate plasma shapes in nonideal traps after the plasma has expanded into a pancake shape. In the following discussion it is assumed that only $C_{4}$ is important to simplify things. In general, the higher-order terms are also important.

For example, in a trap with nonzero $C_{4}$, the plasma shape at $T=0$ would be given by

$z_{p}(r)=\frac{z_{\text {ideal }}(r)}{1-3 C_{4} r^{2} / C_{2}}\left(1+\frac{32 a^{5} C_{4} \epsilon_{0}\left[\frac{2}{3}(r / a)^{2}-\frac{4}{15}\right]}{3 N q}\right)$,

where $z_{\text {ideal }}(r)$ is $z_{p}(r)$ for the ideal quadrupole:

$$
z_{\text {ideal }}(r)=\frac{3 N q \sqrt{1-(r / a)^{2}}}{8 \pi a^{2} C_{2} \epsilon_{0}} .
$$

Careful examination of Eq. (B24) provides an interesting physical picture of what happens to the plasma as $C_{4}$ is changed. If $C_{4}$ is of the same sign as $C_{2}$ (and therefore the same as $q$ ), then increasing the magnitude of $C_{4}$ causes the center of the plasma $(r=0)$ to cave in. The critical point where $\sigma(0)=0$ occurs at

$$
\left|C_{4}\right|=\frac{45 N|q|}{128 \epsilon_{0} a^{5}}
$$

or, defining an average axial half-width,

$$
\left\langle z_{p}\right\rangle=\frac{\int_{0}^{a} n_{\text {cold }}(r) z_{p}(r) r d r}{\int_{0}^{a} n_{\text {cold }} r d r},
$$

the critical point may be expressed as

$$
\frac{C_{4} a^{2}}{C_{2}} \approx \frac{45 \pi}{32} \frac{\left\langle z_{p}\right\rangle}{a} \approx \frac{45 \pi}{32} \alpha .
$$

For $a^{2} C_{4} / C_{2}$ any larger than this, Eq. (B24) is no longer valid, as it implies (at $r=0$ ) negative charge density, or charge of the opposite sign, which would not be confined axially. This probably means that the plasma would form into a ring for $C_{4}$ beyond this limit. Because the plasmas being considered are thin, $\alpha$ is very small $(\alpha \ll 1)$, and Eq. (B28) provides a stringent limit on $C_{4}$.

The reader may have noticed the possibility of a zero in the denominator for $C_{4} r^{2} / C_{2}=1 / 3$. This implies a loss of axial confinement at the outer edge of the profile if $a^{2} C_{4} / C_{2}$ gets as big as $1 / 3$, or $a^{2} C_{4}=C_{2} / 3$. However, it is evident from Eq. (B28) that the critical point at which the plasma is no longer a disk is reached long before this occurs.

If $C_{4}$ and $C_{2}$ have opposite signs, increasing $\left|C_{4}\right|$ has a curious effect. If $\left|C_{4}\right|$ is large enough [of the same order as in Eq. (B28)], then Eq. (B24) implies negative charge density beyond a certain radius. This suggests that as the plasma expands radially and approaches thermal equilibrium in the presence of some nonzero $C_{4}$, it may form into rings around a central disk, or collect on the walls of the trap, as discussed in Sec. A 4.

Some of these physical effects may be seen in the radial density profile of a warm $(T>0)$ plasma as $C_{4}$ (same sign as $C_{2}$ ) is changed. As $\left|C_{4}\right|$ approaches the critical value in Eq. (B28), both the warm and cold plasmas exhibit the same general behavior: the charge density $\sigma(0)$ approaches zero. The cold theory predicts that the plasma will maintain uniform density, thereby being forced to cave in near $r=0$ until it eventually becomes a ring in order to satisfy the condition that $\sigma(0)=0$. The warm plasma, unable to get any thinner than about $\lambda_{\mathrm{D} 0}$, must also satisfy this condition, and it does so by dropping the central density until it, too, has formed a ring. For the $T>0$ case shown in Fig. 5, $C_{4}$ is at about one-twentieth of its critical value, suggesting that if $\left|C_{4}\right|$ were increased by about a factor of 20 , the central density would drop to zero. We have verified this prediction in numerical experiments.

${ }^{1}$ C. S. Weimer, J. J. Bollinger, F. L. Moore, and D. J. Wineland, Phys. Rev. A 49, 3842 (1994)

${ }^{2}$ G. W. Mason, R. L. Spencer, and J. A. Bennett, Phys. Plasmas 3, 1502 (1996).

${ }^{3}$ D. H. E. Dubin, Phys. Rev. Lett. 66, 2076 (1991).

${ }^{4}$ S. A. Prasad and T. M. O’Neil, Phys. Fluids 22, 278 (1979).

${ }^{5}$ R. L. Spencer, S. N. Rasband, and R. R. VanFleet, Phys. Fluids B 5, 4267 (1993).

${ }^{6}$ J. J. Bollinger, D. J. Heinzen, F. L. Moore, Wayne M. Itano, D. J. Wineland, and D. H. E. Dubin, Phys. Rev. A 48, 525 (1993).

${ }^{7}$ D. H. E. Dubin, Phys. Rev. E 53, 5268 (1996).

${ }^{8}$ D. J. Wineland, J. J. Bollinger, W. M. Itano, and J. D. Prestage, J. Opt. Soc. Am. B 2, 1721 (1985).

${ }^{9}$ J. B. Jeffries, S. E. Barlow, and G. H. Dunn, Int. J. Mass Spectrom. Ion Proc. 54, 169 (1983). 